\title{
Sistema Regional de Vacunas (SIREVA), vigilancia por laboratorio y desarrollo de vacunas para Streptococcus pneumoniae: análisis bibliométrico, 1993-2019
}

\author{
José Luis Di Fabio', Clara Inés Agudelo² y Elizabeth Castañeda
}

Forma de citar

Di Fabio JL, Agudelo CI, Castañeda E. Sistema Regional de Vacunas (SIREVA), vigilancia por laboratorio y desarrollo de vacunas para Streptococcus pneumoniae: análisis bibliométrico, 1993-2019. Rev Panam Salud Publica. 2020;44:e80. https://doi.org/10.26633/RPSP.2020.80

RESUMEN Objetivo. Medir a través del análisis bibliométrico la productividad, la visibilidad y el impacto del Sistema
Regional de Vacunas (SIREVA, un proyecto de la Organización Panamericana de la Salud) en sus dos com-
ponentes, la vigilancia por laboratorio y el desarrollo de vacunas.
Métodos. Se recuperaron de Scopus las publicaciones correspondientes a vigilancia por laboratorio y
desarrollo de vacunas con sus referencias y citas y se aplicó la herramienta para obtener los indicadores
bibliométricos. Se utilizó VOSviewer ${ }^{\circledR} 1.6 .13$ para visualizar las redes de coautoría por país y autores, y para
realizar un análisis de coocurrencia de términos incluidos en los títulos y resúmenes de las publicaciones.
Resultados. Cumplieron los criterios establecidos para vigilancia por laboratorio y desarrollo de vacunas 173
y 128 publicaciones, respectivamente. Diez países de la Región fueron responsables de $90,8 \%$ de las publi-
caciones sobre vigilancia por laboratorio y 8 países de todas las publicaciones sobre desarrollo de vacunas.
Las diez publicaciones más citadas sobre vigilancia por laboratorio y sobre desarrollo de vacunas estaban
incluidas en 855 y 503 artículos, respectivamente; los principales autores se encontraban en Alemania, Aus-
tralia, Bélgica, Brasil, China, Estados Unidos de América y el Reino Unido. La construcción y visualización de
redes por coautorías de autores y países, y la coocurrencia de términos mostraron el trabajo en red y la cola-
boración intrarregional, así como permitieron hacer seguimiento a las diversas áreas de estudio y su evolución
en el tiempo. Conclusiones. El análisis bibliométrico permitió documentar de manera objetiva la productividad y visibilidad del Sistema Regional de Vacunas para Streptococcus pneumoniae en la Región.

Palabras clave Streptococcus pneumoniae; vacunas; bibliometría; vigilancia; América Latina.

A finales de los años 80, la Organización Panamericana de la Salud (OPS) consideró la importancia de fortalecer la capacidad científica y tecnológica de los países de América Latina y el Caribe para el desarrollo de los productos inmunobiológicos que se requerían para combatir los problemas prioritarios de salud en la Región de las Américas. Para ello, consideró la conformación del Sistema Regional de Vacunas para América Latina (SIREVA) fundada sobre el amplio espectro de la capacidad científico-técnica de los países para la investigación básica y el desarrollo de vacunas disponible o potencialmente asequible para complementar e integrar los esfuerzos que se realizaban en forma aislada (1).

En esa oportunidad, se decidieron los criterios de selección de las vacunas a ser desarrolladas o mejoradas $y$, para la fase inicial, se propusieron las dirigidas contra las enfermedades producidas por Streptococcus pneumoniae, Salmonella typhi,

\footnotetext{
1 Consultor independiente, Washington D.C., Estados Unidos de América. $\triangle$ difabioj@gmail.com
}

2 Instituto Nacional de Salud, Bogotá, Colombia. 
Neisseria meningitidis grupo B y virus del dengue. En 1992, un grupo de expertos evaluó un plan maestro para una vacuna contra S. pneumoniae. Este plan identificó como prioridad realizar un estudio de la distribución de los serotipos de S. pneumoniae causantes de enfermedad invasora en América Latina y el Caribe que permitiría definir la formulación de una vacuna multivalente adecuada a las características epidemiológicas de la Región (1). En él se planteaba el desarrollo de vacunas conjugadas al igual que la necesidad de incursionar en el tema de vacunas alternativas basadas en las proteínas de S. pneumoniae.

En 1993, con el apoyo financiero del gobierno de Canadá, se inició el estudio de vigilancia por laboratorio de la distribución de serotipos y resistencia antimicrobiana de S. pneumoniae, agente de enfermedad invasiva en niños menores de 5 años.

Después de más de 25 años de conformado SIREVA, se decidió llevar a cabo una medición objetiva del impacto del proyecto tanto en su productividad como en las principales áreas temáticas en que los actores involucrados han estado trabajando. Para ello se realizó un análisis bibliométrico que tuvo por objetivo fundamental el estudio de la producción científica, su evolución y distribución, la dinámica de los grupos que producen y consultan dichos documentos y la información que contienen, sumado a la aplicación de programas de visualización bibliométricos $(2,3)$.

El presente análisis tiene como objetivos mostrar la evolución del proyecto SIREVA en sus dos componentes: el de vigilancia, realizado por los laboratorios de referencia de los países, y el de desarrollo de vacunas. Además, se miden la productividad expresada con base en el número de publicaciones registradas en 27 años (1993-2019) y el impacto que han tenido esas publicaciones, medido por el número de citaciones consignadas en la literatura. Por otra parte, se intentó visualizar interrelaciones entre los profesionales, instituciones y países que participaron en SIREVA durante los años analizados y las principales áreas temáticas de trabajo.

\section{MATERIALES Y MÉTODOS}

\section{Diseño del estudio, estrategia y análisis de los datos}

Realizamos un análisis bibliométrico de documentos publicados entre 1993 y 2019 en revistas indexadas en Scopus e incluimos las siguientes tipologías: artículos originales, artículos de revisión, comunicaciones breves, notas, cartas, editoriales, capítulos de libro y resúmenes en congresos; los idiomas seleccionados fueron español, inglés y portugués.

La búsqueda se realizó en setiembre de 2019. Inicialmente, se seleccionaron, en títulos, resúmenes y palabras clave, los términos: "Streptococcus pneumoniae" y los nombres de los países latinoamericanos. Una siguiente selección utilizó el término serotipo para dirigir la búsqueda a vigilancia por laboratorio y se utilizaron los términos "vacuna" y "desarrollo" para el tema de desarrollo de vacunas (figura 1). Los autores analizaron todos los títulos y eliminaron duplicados y aquellos que no cumplieran con el criterio de búsqueda. Los términos de búsqueda detallados se consignaron en la información suplementaria, cuadro 1. Luego, por selección manual, las publicaciones en la lista de Scopus que cumplían con vigilancia por laboratorio

FIGURA 1. Diagrama de flujo para la recuperación de la bibliografía: vigilancia por laboratorio y desarrollo de vacunas

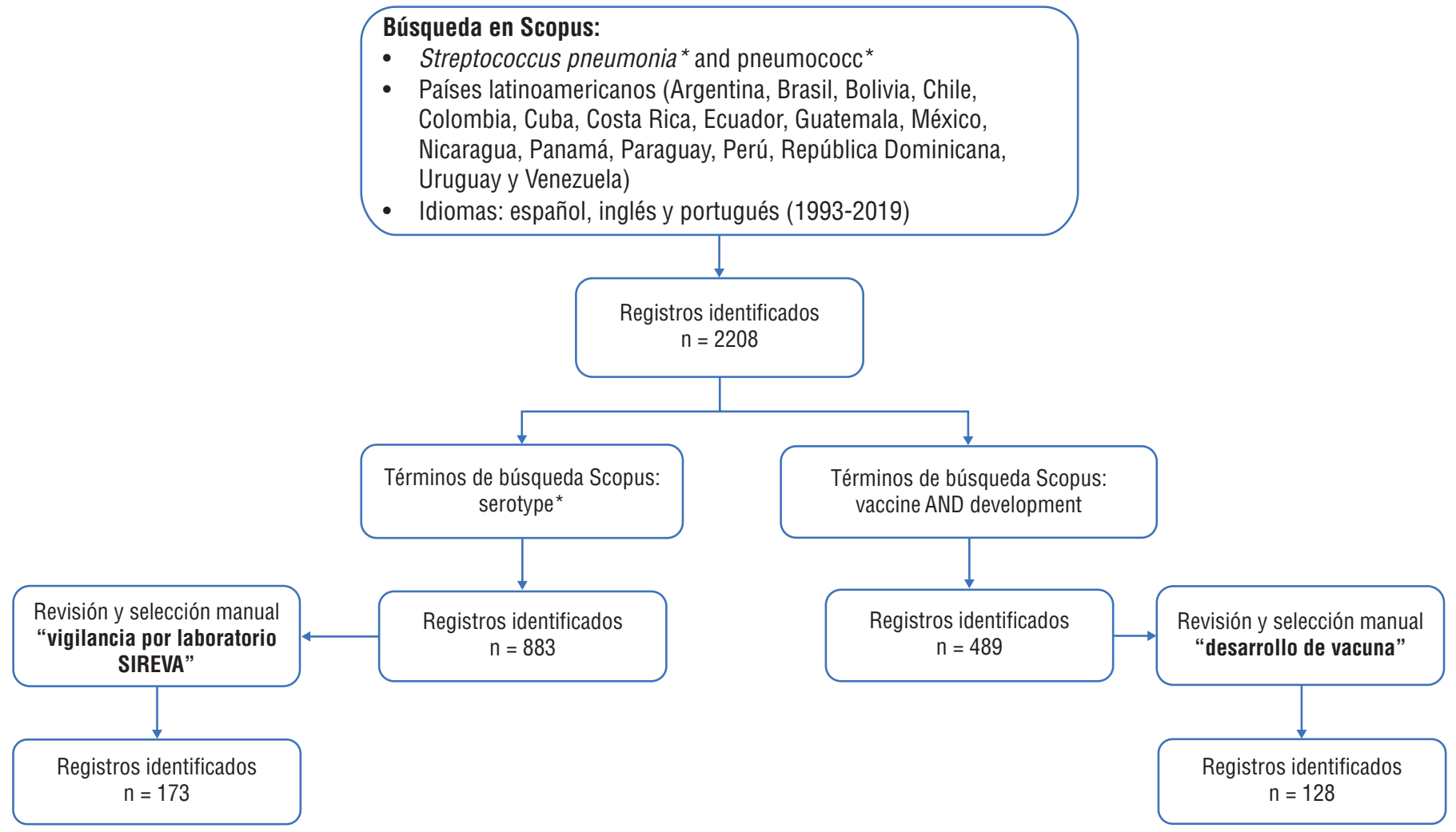


CUADRO 1. Principales publicaciones con base en el mayor número de citaciones, Scopus, 1993-2019

\begin{tabular}{|c|c|c|c|c|c|}
\hline Autor & Revista & Año & citas con autocitas & citas sin autocitas & Ref \\
\hline \multicolumn{6}{|c|}{ Vigilancia por el laboratorio } \\
\hline Park IH, et al. & J Clin Microbiol & 2007 & 348 & 301 & 5 \\
\hline Deeks SL, et al. & Pediatrics & 1999 & 132 & 127 & 7 \\
\hline Di Fabio JL, et al. & Pediatr Infect Dis J & 2001 & 89 & 48 & 8 \\
\hline Kertesz DA, et al. & Clin Infect Dis & 1998 & 80 & 57 & 9 \\
\hline Castañeda $E$, et al. & Pediatr Infect Dis J & 2009 & 65 & 53 & 11 \\
\hline Brandileone MCDC, et al. & Microb Drug Resist & 1997 & 59 & 36 & 12 \\
\hline Lagos $R$, et al & $\mathrm{J}$ Infect Dis & 2008 & 56 & 53 & 13 \\
\hline Vela Coral MC, et al. & Emerg Infect Dis & 2001 & 57 & 26 & 14 \\
\hline \multicolumn{6}{|l|}{ Desarrollo de vacunas } \\
\hline Oliveira MLS, et al. & Microbes Infect & 2006 & 67 & 52 & 18 \\
\hline Racedo S, et al. & Microbes Infect & 2006 & 63 & 32 & 19 \\
\hline Arêas APM, et al. & Biochem Biophys Res Commun & 2004 & 52 & 40 & 20 \\
\hline Rigden DJ, et al. & Crit Rev Biochem Mol Biol & 2003 & 51 & 38 & 21 \\
\hline Miyaji EN, et al. & Cell Mol Life Sci & 2013 & 49 & 44 & 22 \\
\hline Gámez G, et al. & Curr Drug Targets & 2012 & 48 & 28 & 23 \\
\hline Darrieux M, et al. & Crit Rev Microbiol & 2015 & 46 & 35 & 24 \\
\hline
\end{tabular}

Fuente: Scopus

fueron limitadas a las que correspondían a SIREVA o en asociación con SIREVA. De forma similar, las publicaciones que cumplían con el criterio desarrollo de vacunas se limitaron a los artículos que contenían en el título o en el resumen términos asociados con desarrollo de vacunas con base en polisacáridos, proteínas de neumococo, proteínas recombinantes, producción y purificación de polisacáridos y proteínas, modelos animales, respuesta inmunitaria y adyuvantes.

Para el análisis de la información, se utilizaron las propias funciones del menú de Scopus que permite recuperar el número de artículos publicados, por autor, por país, por año, por revista, por tipo de documento y por el total de citas, entre otros. Una herramienta útil de Scopus es que permite la inclusión y exclusión de términos. Esta herramienta fue usada para la selección de los artículos más citados, para luego analizar las citas por artículo eliminando las autocitas y extraer los indicadores bibliométricos seleccionados: número de citas, revistas donde se publicaron y países de los autores que citaron. Para la calidad de las publicaciones, se determinó la clasificación de las revistas con base en índice h y el Scimago Journal Rank (SJR).

\section{Visualización indicadores bibliométricos}

La construcción y visualización de redes bibliométricas fue realizada con el programa VOSviewer ${ }^{\circledR}$ v.1.6.13 para Microsoft Windows (Centre for Science and Technology Studies, Leiden University, The Netherlands) (4). Se seleccionaron los siguientes tipos de redes: a) Redes colaborativas entre países por coautoría, con un mínimo de tres publicaciones por país.

b) Redes de investigadores construidas por coautorías, con un mínimo de dos publicaciones por autor.

c) Redes por coocurrencia de términos en los títulos y en los resúmenes de las publicaciones, con un mínimo de cinco ocurrencias.

Los nodos y vinculaciones en las visualizaciones fueron ajustados por peso, por número de publicaciones y por citaciones. El tamaño de los nodos, la proximidad entre ellos y el grosor de las líneas indican la fuerza de la vinculación.

Los países, autores y términos para las visualizaciones fueron exportados de la herramienta VOSviewer ${ }^{\circledR 3}$ y analizados para normalizarlos posteriormente con el empleo de los tesauros creados: uno de países, uno de autores, para asegurar que los distintos nombres se refirieran al mismo autor y uno de términos para consolidar sinónimos, corregir ortografía, abreviaturas y eliminar términos generales.

\footnotetext{
El lector interesado puede encontrar más información sobre el manejo del VOSviewer $^{\circledR}$, tesauros de autores y términos, composición de las agrupaciones establecidas por el programa para los mapas de autores y términos, y los archivos con la información de los títulos de las publicaciones de vigilancia por laboratorio y desarrollo de vacunas en la web: http:/ / redsirevanetwork.com/ archivos /
} 


\section{Consideraciones éticas}

La información analizada en el trabajo fue extraída de la base de datos Scopus, por lo que no fue necesario contar con aprobación de un comité de ética.

\section{RESULTADOS}

\section{Resultados de la búsqueda}

En la figura 1 se presenta el diagrama de flujo para la recuperación de la bibliografía de los dos temas de análisis. Se identificaron 173 publicaciones sobre vigilancia por laboratorio y 128 en desarrollo de vacunas. El número de documentos publicados por año se presenta en la figura 2.

\section{Análisis de las publicaciones}

Vigilancia por laboratorio. Los 173 artículos sobre vigilancia fueron publicados en 53 revistas. El nombre de la revista, porcentaje de las publicaciones, índice h y el SJR de las cinco revistas principales fueron: Microbial Drug Resistance (10,4\%, 62 y 0,95), Vaccine (8,7\%, 164 y 1,76), Journal of Clinical Microbiology (6,9\%, 234 y 2,31), Revista Panamericana de Salud Pública $(6,9 \%, 51$ y 0,55) y Biomédica $(5,8 \%, 24$ y 0,28). (Información suplementaria, cuadro 2).

Las publicaciones se originaron, en el 90,8\% (157/173) de los casos, en diez países de la Región: Brasil aportó $51(29,5 \%)$, Colombia 33 (19,1\%), Argentina 20 (11,6\%), México 18 (10,4\%), Uruguay $14(8,1 \%)$, Chile $9(5,2 \%)$, Cuba $6(3,5 \%)$, Guatemala 3 $(1,7 \%)$, Venezuela $2(1,2 \%)$ y Perú $1(0,6 \%)$. Los 16 (9,2\%) artículos restantes fueron publicaciones de la Región en conjunto. Las publicaciones con colaboración internacional fueron 39 (22,5\%): Brasil con 16 artículos, Colombia con 6, Argentina, Uruguay, Chile y Guatemala con 3 cada uno, México y Venezuela con 2 cada uno y Perú con 1. (Información suplementaria, cuadro 3).

Las diez publicaciones más citadas, sin autocitas (5-14), que se presentan en el cuadro 1 tuvieron 855 citas en 802 artículos publicados en 260 revistas. El porcentaje, índice h y SJR de las cinco

FIGURA 2. Número de documentos publicados por año SIREVA 1993-2019. A, vigilancia por el laboratorio. B, desarrollo de vacunas

A $\quad \mathrm{n}$

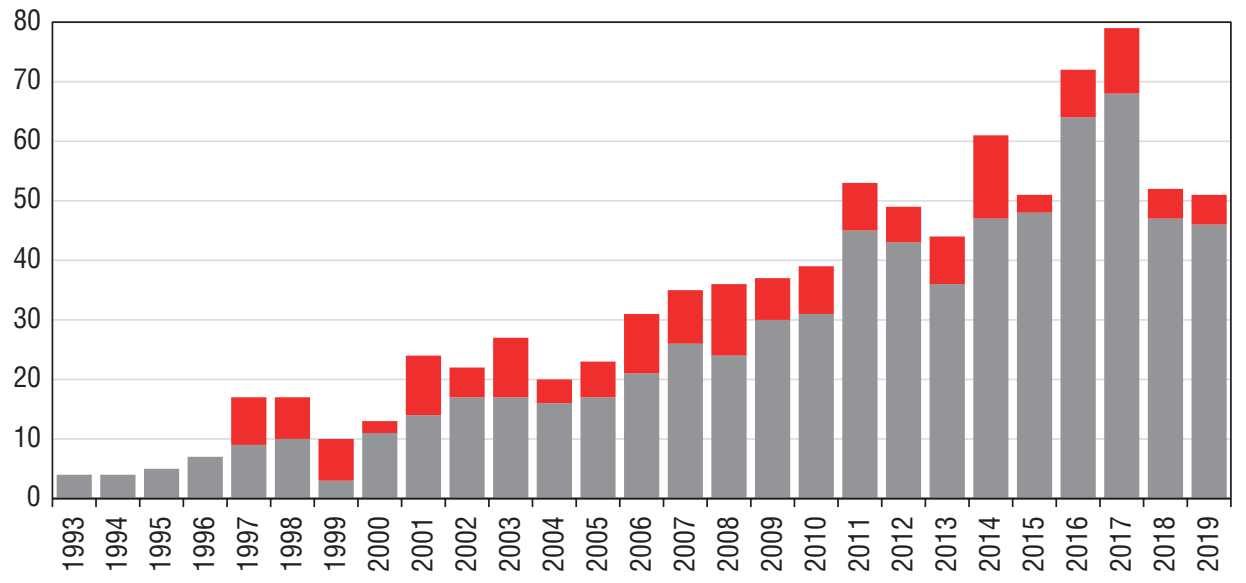

- ("streptococcus pneumonia*" or pneumococc $\left.{ }^{\star}\right)+$ serotype $^{*}+$ vigilancia por laboratorio

- ("streptococcus pneumonia*" or pneumococc $\left.{ }^{\star}\right)+$ serotype* $^{*}$

B $\mathrm{n}$

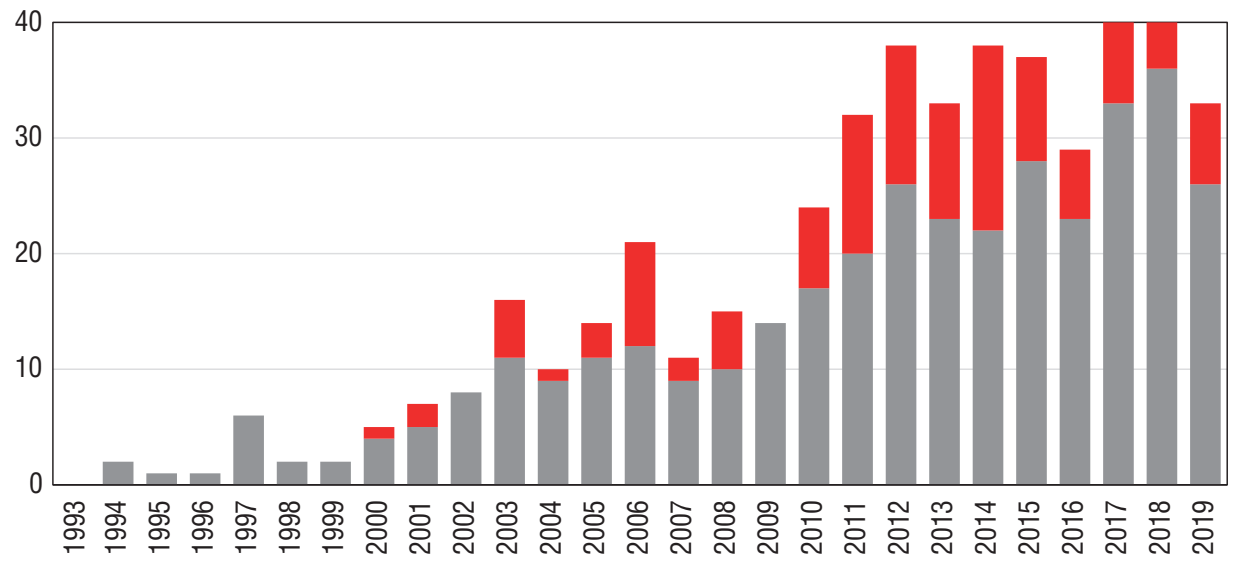

ש ("streptococcus pneumonia*" OR pneumococc* $)+($ vaccine and development) + desarrollo de vacuna

- ("streptococcus pneumonia*" OR pneumococc* $)+$ (vaccine and development) 
FIGURA 3. Diagrama del análisis realizado con VOSviewer. A, mapa de la red colaborativa de coautoría por país de vigilancia por laboratorio. B, diagrama del análisis de autores por coautoría de vigilancia por laboratorio. C, diagrama del análisis de autores por coautoría de desarrollo de vacunas

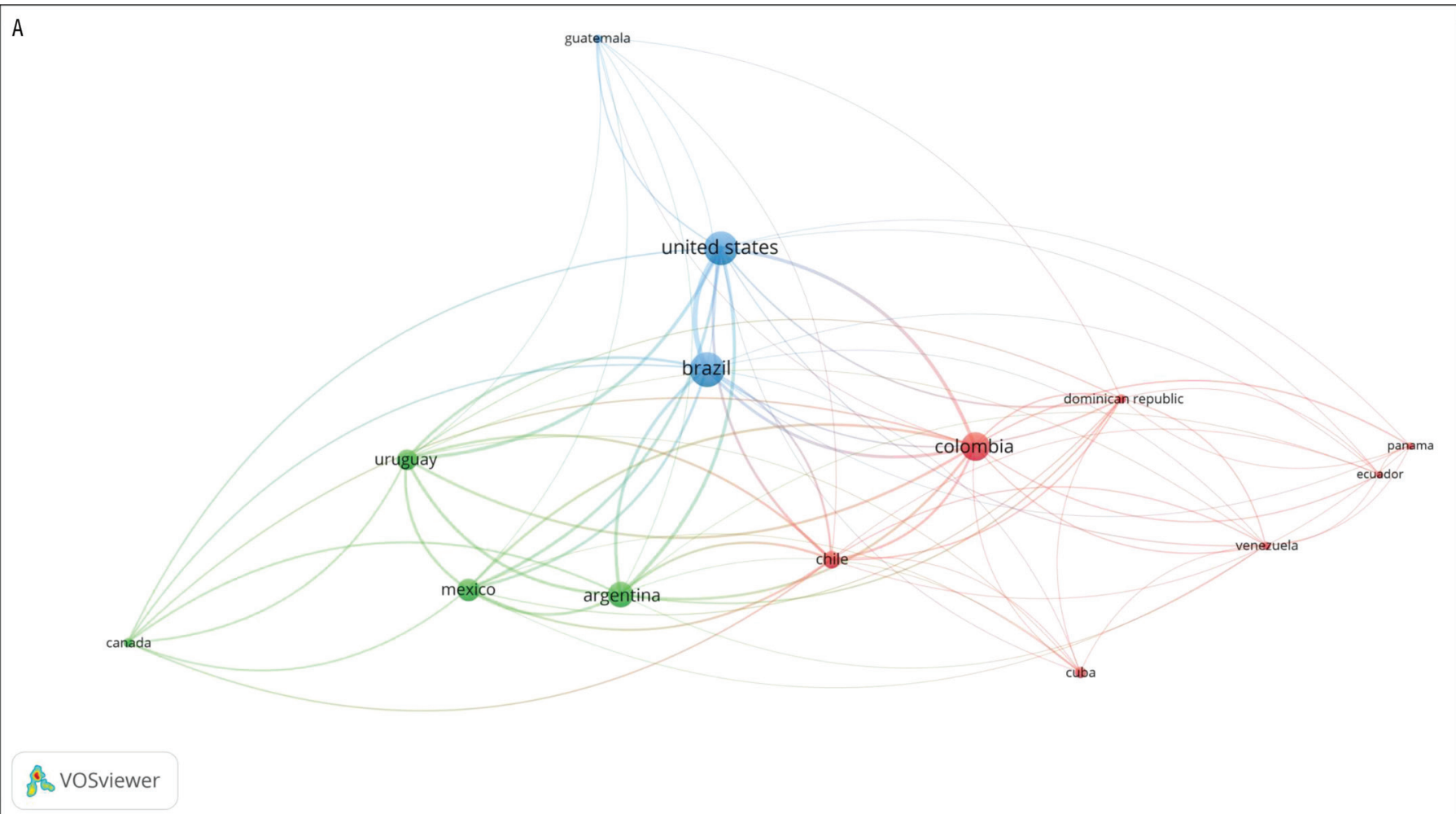

Para la construcción del diagrama del análisis, de los 31 países identificados por VOSviewer, solo 14 países cumplieron con un mínimo de tres documentos por país.

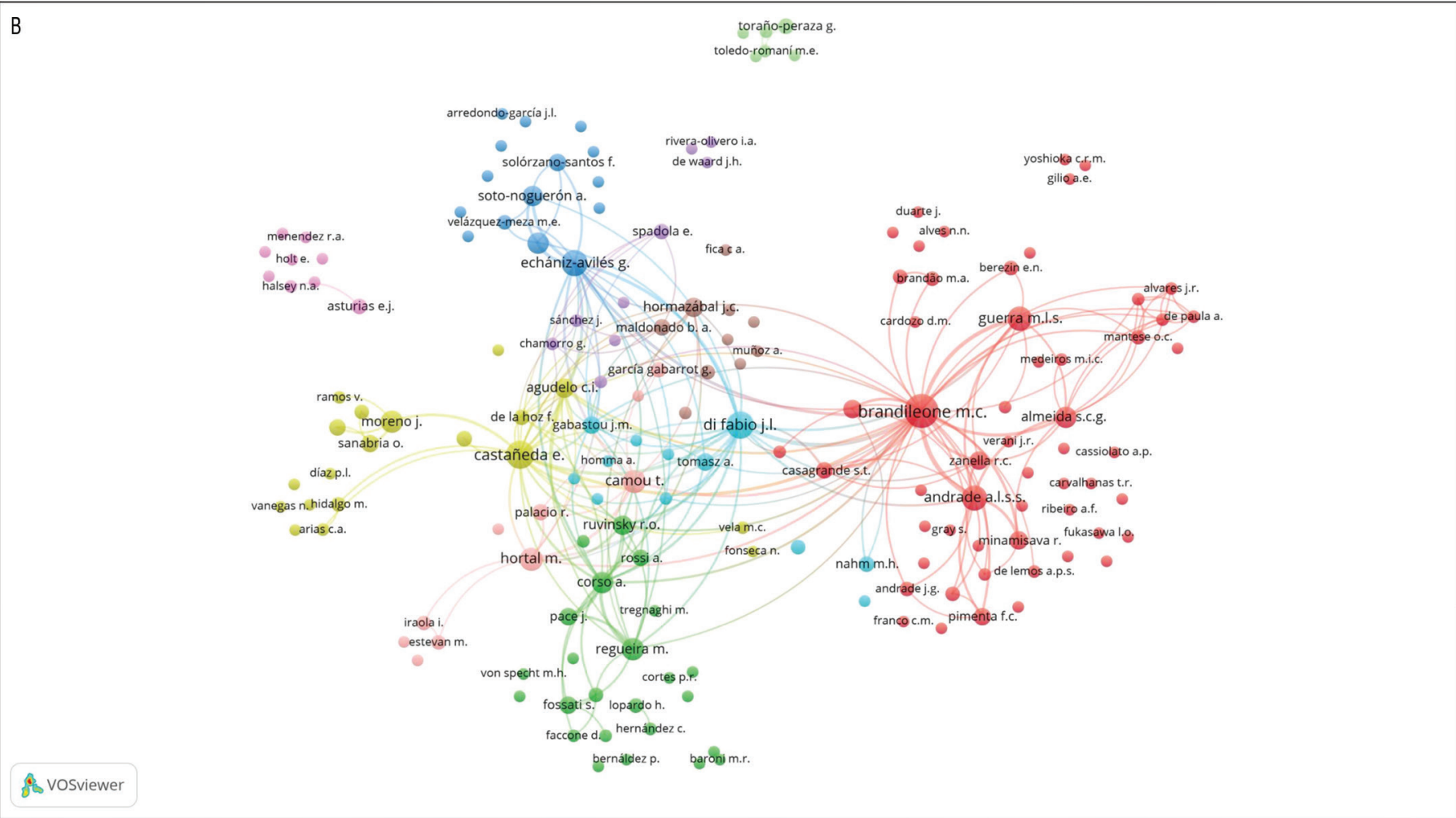

Para la construcción del diagrama VOSviewer identificó 639 autores de los cuales 170 contaban con un mínimo de dos documentos, en el diagrama con 170 nodos se visualizan 10 agrupaciones (información suplementaria 6a): Argentina (verde), Brasil (roja), Colombia (mostaza), México (azul) y Uruguay (rosado). Se distingue también una agrupación de Chile (marrón), una de Guatemala (lila), una que incluye República Dominicana, Venezuela y Paraguay (violeta) y una que representa la coordinación regional o centros de entrenamiento en Canadá y Estados Unidos de América (celeste). La agrupación de Cuba (verde claro), está desvinculada. 


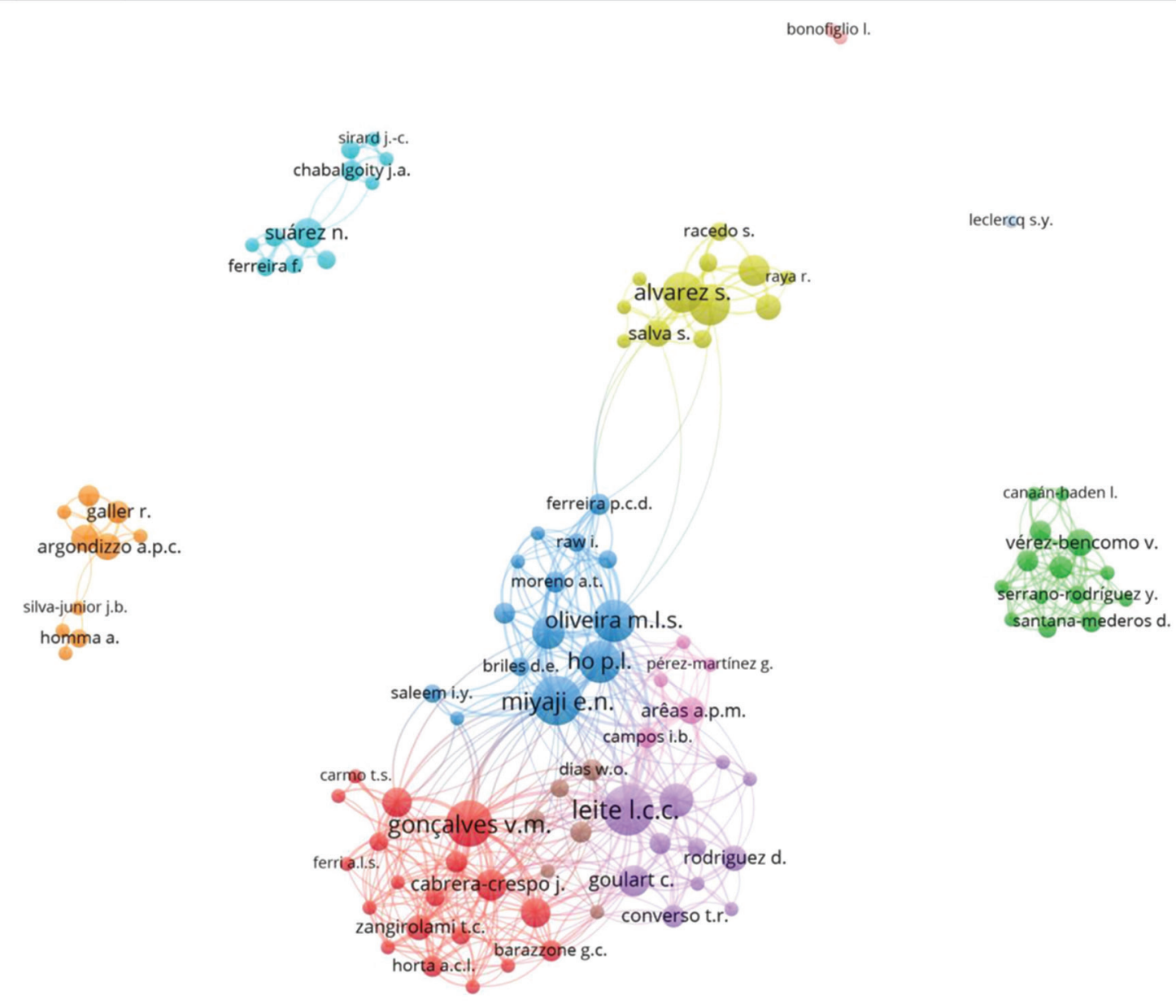

Vosviewer

Para la construcción del diagrama VOSviewer identificó 417 autores de los cuales 107 contaban con un mínimo de dos documentos. En los 107 nodos se visualizan doce agrupaciones, cinco centrales que corresponden a investigadores de los Centros de Biotecnología y Laboratorio de Bioprocesos del Instituto Butantán en San Pablo: una, en el tema de producción y optimización de la producción de polisacáridos capsulares, antígenos proteicos y conjugación (rojo), la segunda en el de vacunas de neumococo basadas en las proteinas de superficie (PspA, pneumolisina) y en la expresión en otros microorganismos (morado), la tercera en la respuesta inmunitaria en animales y uso de las vias de administración nasal e inmunidad de mucosas (rosado), una cuarta en una vacuna a célula completa muerta de neumococo (gris oscuro) y la quinta en la expresión de antigenos en Lactobacillus u otros microorganismos (azul) trabajo vinculado con el Centro de Referencia de Lactobacilos y la Universidad Nacional de Tucumán en Argentina (mostaza). La agrupación de Higiene de la Universidad de la República del Uruguay que se ha concentrado en el tema de producción y purificación de los polisacáridos capsulares y colaboración con Francia. La agrupación correspondiente a Biomanguinhos, Fiocruz (naranja) en Brasil trabaja en el tema antígenos proteicos del neumococo.

publicaciones principales fueron: Vaccine $(8,7 \% ; 164 ; 1,76)$, Journal of Clinical Microbiology $(5,7 \% ; 234 ; 2,31)$, PLOS ONE (4,2\%; 268; 1,10), Pediatric Infectious Disease Journal $(3,7 \% ; 133 ; 1,32)$ y Clinical Infectious Diseases (3,5\%; 303; 4,40). (Información suplementaria, cuadro 4a).

Autores de 89 países publicaron los 802 artículos que referencian a los diez artículos más citados sobre vigilancia por laboratorio: 247 de Estados Unidos, 98 del Reino Unido, 78 de Brasil, 49 de Bélgica, 45 de Australia, 43 de España y 42 de Holanda y 42 de Canadá, entre otros. (Información suplementaria, cuadro $4 b$ ).

Desarrollo de vacunas. Los 128 artículos sobre desarrollo de vacunas fueron publicados en 81 revistas. El título, porcentaje de las publicaciones, índice $\mathrm{h}$ y el SJR de las principales cinco revistas fueron: Vaccine $(9,4 \%, 164$ y 1,76), Clinical and Vaccine Immunology (5,5\%, 98 y 1,40), PLOS ONE (3,9\%, 268 y 1,10), Expert Review of Vaccines $(3,1 \%, 75$ y 1,60) y Microbes and Infection (2,3\%, 129 y 1,14) (información suplementaria, cuadro 2).

Las publicaciones se originaron en ocho países: Brasil con 68 (53,1\%), Argentina con 26 (20,3\%), Uruguay con 11 (8,6\%), Cuba con $10(7,8 \%)$, Colombia con $6(4,7 \%)$, México con $5(3,9 \%)$, Chile con $1(0,8 \%)$ y Venezuela con $1(0,8 \%)$. Las publicaciones en asociación con instituciones de otros países fueron 51 $(39,8 \%)$ : Brasil con 29 artículos, Argentina con 10, Colombia con 4, México con 3, Uruguay y Cuba con 2 cada uno y Chile con 1 (información suplementaria, cuadro 3).

Las diez publicaciones más citadas, sin autocitas (15-24) (cuadro 1), tuvieron 503 citas en 488 artículos publicados en 320 revistas. El título, el porcentaje de publicaciones, el índice $h$ 
FIGURA 4. Coocurrencia de términos en títulos y resúmenes. A, visualización de la coocurrencia. B, visualización temporal de la coocurrencia

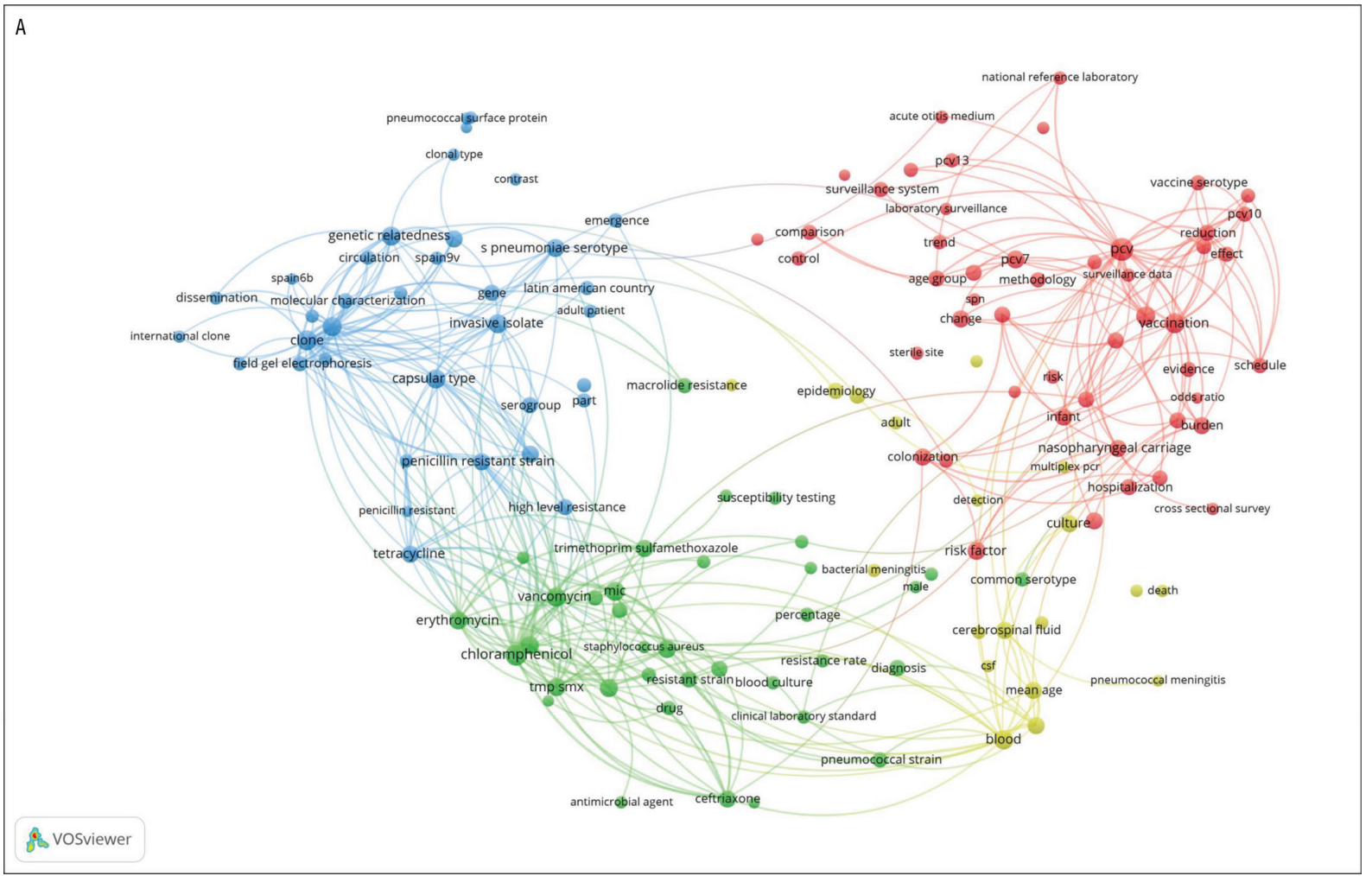

El sistema hizo un análisis de los términos y los ordenó en cuatro agrupaciones, la principal con términos relacionados con vacunación, vacuna de neumococo, PCV7, PCV10, PCV13 (roja). La segunda, con el término resistencia y serotipo, en estrecha asociación con estudios y caracterización genética, estructurales y moleculares, clones y técnicas como PCR y PFGE (azul). En la tercera se destacan términos asociados con resistencia antimicrobiana (concentración inhibitoria mínima) y algunos antibióticos específicos: cloranfenicol, SXT, vancomicina, y otros (verde) y una cuarta que está relacionada con diagnósticos clínicos (mostaza).

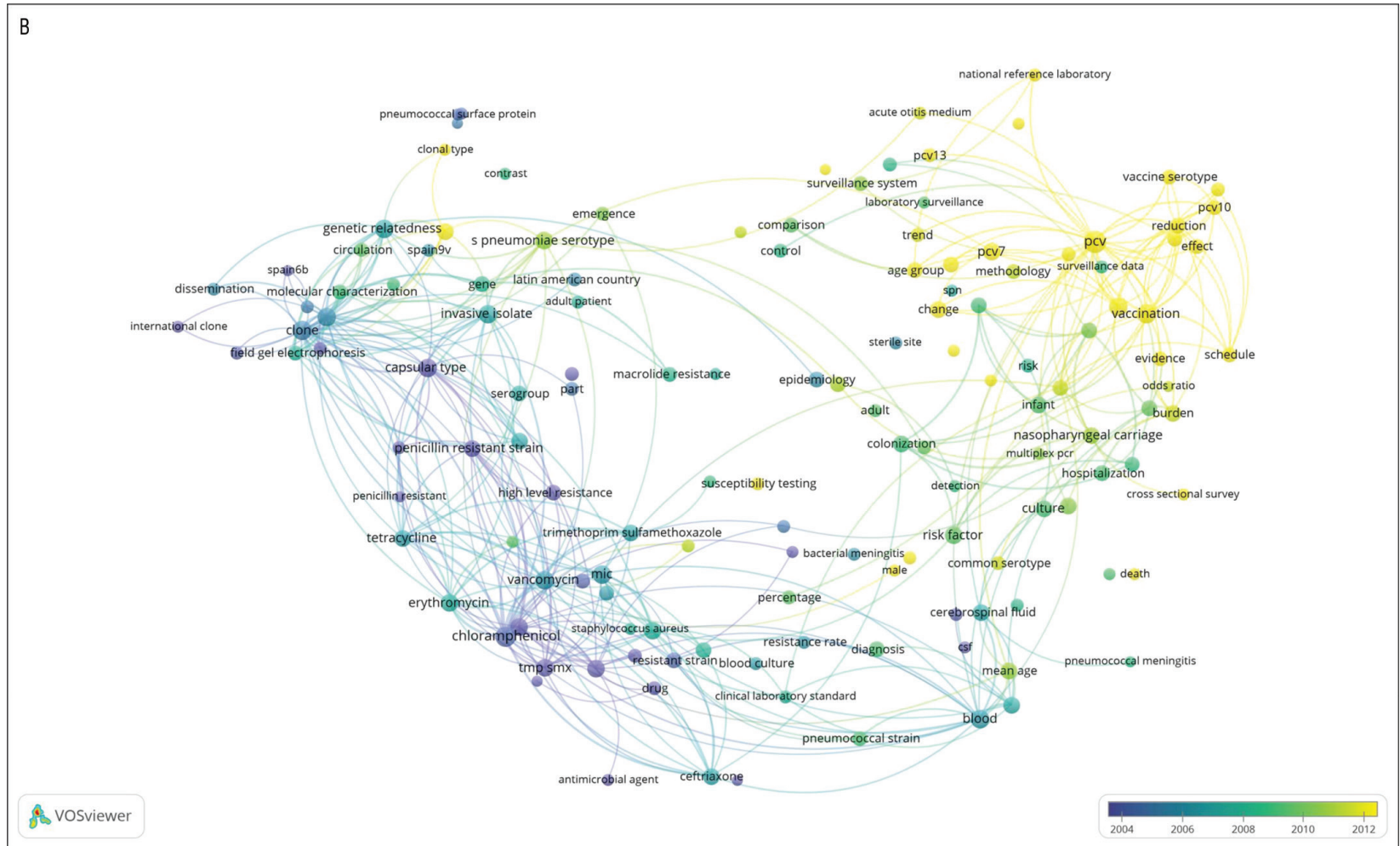

En los mapas de visualización temporal o visualización superpuesta los colores de los nodos indican el año promedio de las publicaciones en que el término fue utilizado, los que se han usado en promedio más recientemente están en amarillo y los empleados en los años anteriores en morado. 
y el SJR de las cinco publicaciones principales fueron; Vaccine $(24 \%, 164,1,76)$, PLOS ONE $(17 \%, 268,1,10)$, Infection and Immunity (15\%, 204, 1,59) (15), Carbohydrate Polymers (8\%, 172 1,38) y Clinical and Vaccine Immunology $(8 \%, 98,1,40)$ (información suplementaria, cuadro 4a). Autores de 61 países publicaron los 488 artículos: 124 de China, 102 de Estados Unidos, 30 del Reino Unido, 30 de Brasil, 27 de Alemania, 26 de Francia, 25 de Irán, 23 de India y 22 de España (información suplementaria, cuadro 4b).

\section{Visualización}

Red colaborativa entre países. El diagrama del análisis de coautoría por país se presenta en la figura 3a. Hay que destacar que el nodo de Estados Unidos incluye 61 documentos, de los cuales 29 incluyen a la OPS y los restantes incluyen a la Universidad Rockefeller en Nueva York, la Universidad de Alabama en Birmingham, los Centros para el Control y Prevención de Enfermedades (CDC) y la Universidad Johns Hopkins en Baltimore.

Autores por coautoría. El diagrama del análisis en el tema vigilancia por laboratorio se presenta en la figura 3b. El trabajo regional se ve marcado por el intenso flujo de vinculaciones hacia el centro del diagrama donde se encuentran autores responsables de coordinación nacional y regional o centros de entrenamiento principalmente en los Estados Unidos de América.

En el diagrama del análisis en el tema de desarrollo de vacunas también se tomaron un mínimo de dos documentos por autor y quedan claramente marcados los grupos involucrados en las investigaciones (figura 3c).

Coocurrencia de términos en títulos y resúmenes. Para la construcción del diagrama del análisis de términos por coocurrencia se tomaron un mínimo de cinco. Del total de 3305 términos que VOSviewer ${ }^{\circledR}$ identificó por conteo binario (presencia o ausencia del término en el documento), fueron preseleccionados 246 que luego se redujeron a los 142 más relevantes. Dado que los términos que dominaron en la selección correspondían a "enfermedad neumocócica" o similares (pneumococcal disease, invasive pneumococcal disease, invasive S. pneumoniae) se decidió eliminarlos por no aportar para el análisis (figura 4a).

Los mapas de visualización temporal o visualización superpuesta se presentan en la figura $4 b$. Según se observa en el diagrama, hubo una evolución de las publicaciones iniciales en que se hace referencia a la serotipificación de los aislamientos y la resistencia antimicrobiana, pasando por el uso de técnicas moleculares, a la incorporación del término vacuna y las publicaciones más recientes hacen referencia al uso de las vacunas conjugadas, su evaluación y efectividad.

\section{DISCUSIÓN}

SIREVA planteó como elemento inicial y fundamental obtener datos de vigilancia por laboratorio para patógenos seleccionados. Veinticinco años después la productividad del proyecto en su componente $S$. pneumoniae, está ampliamente documentada en la literatura científica $(7,8,10,25)$; sin embargo, no se había realizado un análisis objetivo de esa productividad y su impacto.

La bibliometría es una herramienta que permite llevar a cabo, de forma objetiva, una evaluación de la actividad y productividad científica $(2,3,26,27)$, por lo que se decidió aplicarla en este estudio donde documentamos la evolución del proyecto SIREVA de la OPS con los países de la Región en los dos componentes seleccionados. El análisis lo realizamos a partir de una búsqueda en la base de datos de Scopus, la base de datos más completa, que incluye el PubMed y permite obtener información detallada de los artículos, sus referencias y citas. Sin embargo, tiene la desventaja de no incluir artículos de revistas con revisión por pares que no están indexadas. Los criterios de búsqueda establecidos y la revisión posterior de todos los títulos obtenidos garantizó la calidad de los datos. Vale la pena mencionar que los tres autores formaron parte activa de SIREVA, lo cual garantizó la fidelidad del dato.

Además, asociamos la búsqueda con el VOSviewer ${ }^{\circledR}$, lo que permitió la construcción y visualización de redes por coautorías y coocurrencia con base en los términos extraídos de los títulos y resúmenes de los artículos. Con esta visualización del conocimiento, se logró hacer el seguimiento de la evolución y cómo se ha encauzado en las diversas áreas dentro del tema de estudio (7-10, 28).

El análisis del número de documentos publicados por año reveló un incremento exponencial de ellos en los dos temas seleccionados para un total de 173 y 128 respectivamente (figura 2). Vale la pena destacar que de 1997 al 2003, los datos iniciales del programa de vigilancia por laboratorio se constituyeron en los primeros datos publicados en la Región sobre el neumococo $(43,3 \%)$ y, a partir de ellos, la mayoría de los países integrantes conformaron grupos de investigación sobre el tema, exitosos, a juzgar por su productividad (figura 3b).

La posibilidad de documentar que más de $80 \%$ de los documentos fueron artículos originales publicados en revistas indexadas con revisión por pares habla de la calidad del dato y garantizó la citación. Para vigilancia por laboratorio, 38,7\% de los artículos fueron publicados en cinco revistas de alto impacto como lo indican sus índices h y SJR, mientras que, para desarrollo de vacunas, $24,4 \%$ fueron publicados en cinco revistas reconocidas en el campo. Sobre el tema vigilancia por laboratorio, 90\% de las publicaciones se originaron en diez países de la Región, con participación entre países y asociadas con entidades internacionales; esto refleja el cumplimiento del trabajo en red, objetivo de SIREVA para esta actividad. Sobre el desarrollo de vacunas, la totalidad de las publicaciones fueron de ocho países, dos de ellos que tenían la capacidad de producción instalada, el Instituto Butantán y Fiocruz en Brasil, y el Instituto de Vacunas Finlay de Cuba (18, 20, 22, 24).

Al analizar las citas de las publicaciones más citadas como indicador de la relevancia de la actividad científica regional en los temas de vigilancia por laboratorio y desarrollo de vacuna para S. pneumoniae y con la información de los autores y las revistas, queda claro que ambos proyectos han tenido un impacto global, con 855 citas y autores, en orden decreciente, de Estados Unidos, Reino Unido, Brasil, Bélgica y Australia principalmente, y 503 citas de autores, en orden decreciente, de China, Estados Unidos, Reino Unido, Brasil y Alemania para cada uno de los temas, respectivamente

Es importante mencionar que la vigilancia por laboratorio contó, a través de la OPS, con recursos financieros, lo que permitió una coordinación eficiente de la red de laboratorios nacionales de referencia. Sin embargo, para el tema de desarrollo de vacunas, la OPS solo facilitó algunas reuniones donde 
convocó a investigadores de la Región a discutir propuestas de colaboración. Los investigadores convocados y otros más desarrollaron luego sus programas de investigación y desarrollo en forma independiente, y las publicaciones relacionadas comenzaron a observarse después del año 2000.

El mapeo y visualización con VOSviewer ${ }^{\circledR}$ establece de forma automática los nodos, vínculos y agrupaciones que constituyen una red y producen resultados objetivos, aunque el sistema permite realizar ajustes manuales que fueron llevados a cabo en nuestro análisis. Los nodos representan los objetos de estudio, por ejemplo, autores, publicaciones o términos; aquellos nodos con mayor peso se muestran más prominentes que los de menor peso. El tamaño del nodo puede reflejar el número de publicaciones del autor, o el número de citas de sus publicaciones o el número de ocurrencias de un término.

El mapa de la red colaborativa de vigilancia por laboratorio SIREVA ilustra la intensa vinculación entre los doce países y los centros de entrenamiento en Canadá y Estados Unidos y la coordinación de la OPS (figura 3a) (7-10, 29-32).

En la visualización por coautoría quedaron definidas 10 agrupaciones correspondientes a los grupos intrapaís, por su colaboración y vinculación con los laboratorios de referencia nacionales representados por los coordinadores de la vigilancia en cada uno de ellos (figura $3 b$ ).

En la visualización por coautoría en desarrollo de vacunas se destacan las cinco agrupaciones correspondientes a investigadores de los Centros de Biotecnología y Laboratorio de Bioprocesos del Instituto Butantán en San Pablo, Brasil, que de manera holística abordaron los diferentes enfoques para el desarrollo de vacuna neumocócica: vacunas conjugadas, vacunas proteicas, vacunas recombinantes, así como estudios inmunológicos y preclínicos $(18,20,22,24)$. Se destacan también la agrupación correspondiente a Cuba con el desarrollo de una vacuna conjugada que ya se encuentra en las fases finales de los estudios clínicos correspondientes (33) y la de Uruguay, que concentró sus esfuerzos en los polisacáridos capsulares de neumococo (15) (figura 3c).

La visualización superpuesta o temporal reveló con claridad la evolución de los temas de investigación en el terreno de $S$. pneumoniae en la Región, probablemente similar a lo que ocurre a nivel global (figuras $4 \mathrm{a}$ y b). Los términos relacionados con tipos capsulares, antibióticos, sensibilidad antimicrobiana, concentración inhibitoria mínima (CIM), resistencia de nivel alto e intermedio, se asocian con las publicaciones de los años 90 $(6-8,10-12)$, seguidas por términos relacionados con clones, electroforesis por campo pulsado (PFGE, por su sigla en inglés), relaciones genéticas $(9,14)$ y continúan con términos usados en publicaciones más recientes como tipificación multilocus de secuencias (MLST, por su sigla en inglés), reacción en cadena de la polimerasa (PCR, por su sigla en inglés), portadores, vacunas, vacunas conjugadas (PCV) y los términos nuevos como son PCV7, PCV10 y PCV13, evaluación, efectividad, y reducción $(13,28,34,35)$.

Se puede ver también una evolución en las metodologías usadas: CIM, PFGE, MLST y PCR. Por supuesto, esta información no es nueva, ni inesperada, pero es interesante ver como los métodos bibliométricos permiten visualizar esta evolución.

Los resultados del análisis y la visualización bibliométrica se pueden considerar como una revisión sistemática de la productividad y visibilidad del trabajo investigativo de los autores y sus redes, con la productividad expresada por el número de publicaciones realizadas en los últimos 27 años para la vigilancia y el desarrollo de vacunas y la visibilidad e impacto por el número de citaciones recibidas y el origen de estas citaciones. SIREVA en su componente S. pneumoniae es, sin lugar a duda, uno de los proyectos más exitosos coordinados por la OPS en los últimos 25 años.

Contribución de los autores. JLDF fue responsable de la concepción del trabajo y obtención de la información de la base de datos. Los tres autores, JLDF, EC y CIA trabajaron en el diseño, análisis e interpretación de los resultados y redacción y revisión del manuscrito, y asumen la responsabilidad de todos los aspectos del trabajo.

Conflicto de intereses. Ninguno declarado por los autores.

Declaración. Las opiniones expresadas en este manuscrito son responsabilidad de los autores y no reflejan necesariamente los criterios ni la política de la RPSP/PAJPH y / o de la OPS.

\section{REFERENCIAS}

1. Sistema Regional de Vacunas para América Latina y el Caribe (SIREVA). XXIX Reunión del Comité Asesor de Investigaciones en Salud, Organización Panamericana de la Salud (OPS). Washington D.C.: OPS; 1993. Disponible en: http://hist.library. paho.org/Spanish/CAIS/14191.pdf Acceso el 23 de enero de 2020.

2. González de Dios J, Moya M, Mateos Hernández MA. Indicadores bibliométricos: Características y limitaciones en el análisis de la actividad científica. An Esp Pediatr. 1997;47(3):235-44.

3. Spinak E. Indicadores cienciométricos. Ci Inf (Brasilia). 1998; 27(2):141-8. Doi: http://dx.doi.org/10.1590/S0100-19651998000200006 Acceso el 12 de mayo de 2020.

4. van Eck NJ, Waltman L. Software survey: VOSviewer, a computer program for bibliometric mapping. Scientometrics. 2010;84(2):523-8. PMID: 20585380
5. Park IH, Pritchard DG, Cartee R, Brandao A, Brandileone MCC, Nahm MH. Discovery of a new capsular serotype (6C) within serogroup 6 of Streptococcus pneumoniae. J Clin Microbiol. 2007;45(4):1225-33.

6. Domingues CM, Verani JR, Montenegro Renoiner EI, de Cunto Brandileone MC, Flannery B, de Oliveira LH, Santos JB, de Moraes JC, and Brazilian Pneumococcal ConjugateVaccine Effectiveness Study Group. Effectiveness of ten-valent pneumococcal conjugate vaccine against invasive pneumococcal disease in Brazil: a matched case-control study. Lancet Respir Med. 2014;2(6):464-71. doi: 10.1016/S2213-2600(14)70060-8.

7. Deeks SL, Palacio R, Ruvinsky R, Kertesz DA, Hortal M, Rossi A, et al. Risk factors and course of illness among children with invasive penicillin-resistant Streptococcus pneumoniae. Pediatrics. 1999;103(2):409-13. 
8. Di Fabio JL, Castañeda E, Agudelo CI, De La Hoz F, Hortal M, Camou T, et al. Evolution of Streptococcus pneumoniae serotypes and penicillin susceptibility in Latin America, Sireva-Vigía Group, 1993 to 1999. Pediatr Infect Dis J. 2001;20(10):959-67.

9. Kertesz DA, Di Fabio JL, De Cunto Brandileone MC, Castañeda E, Echániz-Aviles G, Heitmann I, et al. Invasive Streptococcus pneumoniae infection in Latin American children: results of the Pan American Health Organization surveillance study. Clin Infect Dis. 1998;26(6):1355-61.

10. Tomasz A, Corso A, Severina EP, Echániz-Aviles G, De Cunto Brandileone MC, Camou T, et al. Molecular epidemiologic characterization of penicillin-resistant Streptococcus pneumoniae invasive pediatric isolates recovered in six Latin- American countries: an overview. Microb Drug Resist. 1998;4(3):195-207.

11. Castañeda E, Agudelo CI, Regueira M, Corso A, De Cunto Brandileone MC, Brandão AP, et al. Laboratory-based surveillance of Streptococcus pneumoniae invasive disease in children in 10 Latin American countries: A SIREVA II project, 2000-2005. Pediatr Infect Dis J 2009;28(9):e265-e270.

12. Brandileone MCDC, Vieira VSD, Casagrande ST, Zanella RC, Guerra MLLS, Bokermann S, et al. Prevalence of serotypes and antimicrobial resistance of Streptococcus pneumoniae strains isolated from Brazilian children with invasive infections. Microb Drug Resist. 1997;3(2):141-6.

13. Lagos R, Muñoz A, San Martin O, Maldonado A, Hormazabal JC, Blackwelder WC, et al. Age- and serotype-specific pediatric invasive pneumococcal disease: insights from systematic surveillance in Santiago, Chile, 1994-2007. J Infect Dis 2008;198(12):1809-17.

14. Vela Coral MC, Fonseca N, Castañeda E, Di Fabio JL, Hollingshead SK, Briles DE. Pneumococcal surface protein A of invasive Streptococcus pneumoniae isolates from Colombian children. Emerg Infect Dis. 2001;7(5):832-6.

15. Cuesta G, Suarez N, Bessio MI, Ferreira F, Massaldi H. Quantitative determination of pneumococcal capsular polysaccharide serotype 14 using a modification of phenol-sulfuric acid method. J Microbiol Methods. 2003;52(1):69-73.

16. Ingolotti M, Kawalekar O, Shedlock DJ, Muthumani K, Weiner DB. DNA vaccines for targeting bacterial infections. Expert Rev Vaccines. 2010;9(7):747-63.

17. Muñoz N, Van Maele L, Marqués JM, Rial A, Sirard J-, Chabalgoity JA. Mucosal administration of flagellin protects mice from Streptococcus pneumoniae lung infection. Infect Immun. 2010;78(10):4226-33.

18. Oliveira MLS, Arêas APM, Campos IB, Monedero V, Perez-Martínez G, Miyaji EN, et al. Induction of systemic and mucosal immune response and decrease in Streptococcus pneumoniae colonization by nasal inoculation of mice with recombinant lactic acid bacteria expressing pneumococcal surface antigen A. Microbes Infect. 2006;8(4):1016-24.

19. RacedoS, Villena J, Medina M, Agüero G, Rodríguez V, Alvarez S. Lactobacillus casei administration reduces lung injuries in a Streptococcus pneumoniae infection in mice. Microbes Infect. 2006;8(9-10):2359-66.

20. Arêas APM, Oliveira MLS, Miyaji EN, Leite LCC, Araújo Aires K, Dias WO, et al. Expression and characterization of cholera toxin B-pneumococcal surface adhesin a fusion protein in Escherichia coli: ability of CTB-PsaA to induce humoral immune response in mice. Biochem Biophys Res Commun. 2004;321(1):192-6.

21. Rigden DJ, Galperin MY, Jedrzejas MJ. Analysis of structure and function of putative surface-exposed proteins encoded in the Streptococcus pneumoniae genome: A bioinformatics-based approach to vaccine and drug design. Crit Rev Biochem Mol Biol. 2003;38(2):143-68.

22. Miyaji EN, Oliveira MLS, Carvalho E, Ho PL. Serotype-independent pneumococcal vaccines. Cell Mol Life Sci. 2013;70(18):3303-26.

23. Gámez G, Hammerschmidt S. Combat pneumococcal infections: adhesins as candidates for protein- based vaccine development. Curr Drug Targets. 2012;13(3):323-37.
24. Darrieux M, Goulart C, Briles D, Leite LCDC. Current status and perspectives on protein-based pneumococcal vaccines. Crit Rev Microbiol. 2015;41(2):190-200.

25. Sistema Regional de Vacunas (SIREVA) II, Organización Panamericana de la Salud (OPS). Washington D.C.: OPS. Disponible en: www.paho.org/hq/index.php?option $=$ com_content\&view $=$ article\&id=5536:2011-sireva-ii\&Itemid=3966\&lang=frS Acceso el 23 de enero de 2020.

26. Sweileh WM. Global research trends of World Health Organization's top eight emerging pathogens. Global Health. 2017;13(1):9. Doi: $10.1186 /$ s12992-017-0233-9

27. Rodrigues SP, van Eck NJ, Waltman L, et al. Mapping patient safety: a large-scale literature review using bibliometric visualisation techniques. BMJ Open. 2014;4:e004468. Doi:10.1136/ bmjopen-2013-004468

28. Brandileone MC, Almeida SCG, Minamisava R, Andrade AL. Distribution of invasive Streptococcus pneumoniae serotypes before and 5 years after the introduction of 10-valent pneumococcal conjugate vaccine in Brazil. Vaccine. 2018;36(19):2559-66. Doi: 10.1016/j. vaccine.2018.04.010

29. Garcia S, Levine OS, Cherian T, Gabastou JM, Andrus J, Flannery $\mathrm{B}$, et al. Pneumococcal disease and vaccination in the Americas: An agenda for accelerated vaccine introduction. Rev Panam Salud Publica. 2006;19(5):340-8. Doi: 10.1590/S1020-49892006000500007

30. Gabastou J, Agudelo CI, De Cunto Brandileone MC, Castañeda E, De Lemos APS, Di Fabio JL. Characterization of invasive isolates of S. pneumoniae, H. influenzae, and N. meningitidis in Latin America and the Caribbean: SIREVA II, 2000-2005. Rev Panam Salud Publica. 2008;24(1):1-15. Doi: 10.1590/S1020-49892008000700001

31. Firacative C, Moreno J, Resales P, Maldonado A, Sánchez J, Pesantes C, et al. Circulation of Streptococcus pneumoniae clone Colombia5 ST289 in nine Latin American countries. Rev Panam Salud Publica. 2009;25(4):337-43. Doi: 10.1590/S1020-49892009000400008

32. Lovgren M, Talbot JA, Brandileone MC, Casagrande ST, Agudelo CI, Castañeda E, et al. SIREVA Study Group. Evolution of an international external quality assurance model to support laboratory investigation of Streptococcus pneumoniae, developed for the SIREVA project in Latin America, from 1993 to 2005. J Clin Microbiol. 2007;45(10):3184-90

33. Linares-Pérez N, Toledo-Romaní ME, Casanova González MF, Paredes Moreno B, Váldes Balbín Y, Santana Mederos D, et al. New Cuban pneumococcal vaccine, from available scientific evidence to the strategy of clinical and impact evaluation. Revista Cubana de Pediatria. 2017;89:181-96.

34. Parra EL, De La Hoz F, Díaz PL, Sanabria O, Realpe ME, Moreno J. Changes in Streptococcus pneumoniae serotype distribution in invasive disease and nasopharyngeal carriage after the heptavalent pneumococcal conjugate vaccine introduction in Bogotá, Colombia.Vaccine.2013;31(37):4033-8.Doi:10.1016/j.vaccine.2013. 04.074

35. Echaniz-Aviles G, Soto-Nogueron A, Miranda-Novales G, Carnalla-Barajas MN, Velazquez-Meza ME, Solórzano-Santos F; SIREVA Network-Mexico. Streptococcus pneumoniae serotypes identified in Mexican children with invasive disease before and after the introduction of PCV7 (1993-2012). Arch Med Res. 2015;46(2):149-53. Doi: 10.1016/j.arcmed.2015.02.004mexico

Manuscrito recibido el 17 de marzo de 2020. Aceptado para su publicación, tras revisión, el 14 de mayo de 2020 


\section{Regional System for Vaccines (SIREVA), laboratory surveillance and vaccine development for Streptococcus pneumoniae: bibliometric analysis, 1993-2019}

ABSTRACT Objective. To measure through bibliometric analysis the productivity, visibility and impact of the Regional System for Vaccines (SIREVA, a project by the Pan American Health Organization), including its two components laboratory surveillance and vaccine development.

Methods. Publications about laboratory surveillance and vaccine development were recovered from Scopus, including their references and citations, and their bibliometric indicators were analyzed. VOSviewer 1.6.13 ${ }^{\circledR}$ was used to visualize the co-authorship networks, by country and authors, and to perform a cooccurrence analysis of terms included in the titles and abstracts of the publications.

Results. The criteria for laboratory surveillance and vaccine development were met by 173 and 128 publications, respectively. Ten countries in the Region of the Americas were responsible for $90.8 \%$ of the publications on laboratory surveillance and 8 for all publications on vaccine development. The 10 most cited publications on laboratory surveillance and on vaccine development were included in 855 and 503 articles, respectively, the main authors being from Australia, Belgium, Brazil, China, Germany, the United Kingdom and the United States of America. Network building and visualization by author and country co-authors, and co-occurrence of terms showed networking and intraregional collaboration, and allowed for the follow-up of study areas and evolution over time.

Conclusions. The bibliometric analysis allowed to objectively record the productivity and visibility of the Regional System for Vaccines for Streptococcus pneumoniae in the Region.

Keywords $\quad$ Streptococcus pneumoniae; vaccines; bibliometrics; surveillance; Latin America. 